PERSPECTIVE

\title{
Mammals fail to regenerate organs when wound contraction
} drives scar formation

\author{
loannis V. Yannas $\mathbb{I D}^{1 凶}$ and Dimitrios S. Tzeranis ${ }^{2}$
}

To understand why mammals generally do not regenerate injured organs, we considered the exceptional case of spontaneous skin regeneration in the early lamb fetus. Whereas during the early fetal stage skin wounds heal by regeneration, in the late fetal stage, and after birth, skin wounds close instead by scar formation. We review independent evidence that this switch in wound healing response coincides with the onset of wound contraction, which is also enabled during late fetal gestation. The crucial role of wound contraction in determining the wound healing outcome in adults has been demonstrated in three mammalian models of severe injury (excised guinea pig skin, transected rat sciatic nerve, excised rabbit conjunctival stroma) where grafting the injury with DRT, a contraction-blocking scaffold of highly-specific structure, altered significantly the wound healing outcome. While spontaneous healing resulted in scar formation in these animal models, DRT grafting significantly reduced the extent of wound contraction, prevented scar synthesis, and resulted in partial regeneration. These findings, as well as independent data from species that heal spontaneously via regeneration, point to a striking hypothesis: The process of regeneration lies dormant in mammals until appropriately activated by injury. In spontaneous wound healing of the late fetus and in adult mammals, wound contraction impedes such endogenous regeneration mechanisms. However, engineered treatments, such as DRT, that block wound contraction can cancel its effects and favor wound healing by regeneration instead of scar formation.

npj Regenerative Medicine (2021)6:39; https://doi.org/10.1038/s41536-021-00149-9

\section{INTRODUCTION}

Mammals in their early fetal stage have been shown capable of regenerating skin following severe injury ${ }^{1,2}$. Investigators have been impressed by fetal wound healing observed in several mammalian species, and have occasionally referred to it as "flawless" while others have described the process as "resembling regeneration". However, at about two-thirds of gestation time (late fetal stage) the mammalian fetus experiences a transition from scarless skin wound healing to healing that leads to scar formation $^{3-6}$. Beyond that fetal transition, and for the remainder of the organism's life, skin wounds heal in the standard adult mode, i.e. by scar formation; spontaneous regeneration of adult skin is not observed. With very few exceptions, e.g. rabbit ears ${ }^{7}$ or children's digits ${ }^{8}$, adult mammals fail to spontaneously regenerate skin or any of their injured organs. In sharp contrast to the late mammalian fetus or adult mammals, several amphibians can regenerate entire limbs spontaneously throughout their lifetime ${ }^{9}$.

Investigators have tried for many years to understand how the early mammalian fetus heals without scar in order to apply this knowledge towards functional restoration in diseased organs in humans ${ }^{1-3,10}$. However, the reason behind the loss of regenerative ability during gestation remains unknown. Today it is widely believed that the ability to spontaneously regenerate limbs or organs is an intrinsic property of mammals that cannot be changed later in life.

This article summarizes experimental evidence from fetal wound healing as well as from three adult mammalian injury models where partial organ regeneration was induced by scaffolds of highly specific properties. Evidence is also marshaled from exceptional mammalian species that can spontaneously heal injuries via regeneration in the absence of wound contraction. Combined together, the evidence suggests a key role for wound contraction in impeding spontaneous regeneration and leading instead to scar formation ${ }^{11}$. We hypothesize that the early fetal competence to heal without scar lies dormant in adult mammals and, once reactivated by proper treatments that block wound contraction, can induce regeneration in adult injured or diseased organs.

\section{WOUND CONTRACTION IS ENABLED DURING GESTATION}

In adult mammals, it is widely recognized that the normal macroscopic forces of wound contraction in skin injuries are generated by contractile cells including myofibroblasts (MFB) ${ }^{12,13}$. Indeed, immunostaining for alpha-smooth muscle actin (aSMA; major MFB marker) appears in adult rat skin wounds about 4 days after injury and disappears, due to MFB apoptosis, about 25 days later $^{14}$.

Apart from adult wounds, MFBs appear to play a significant contractile role in fetal wounds. Motivated by the earlier discovery that fetal skin wound healing switches from scarless healing to scar formation during gestation, Estes and coworkers asked whether this transition was related to the extent of MFB differentiation observed in fetal wounds ${ }^{15}$. Indeed, a significant increase in aSMA staining was observed in fetal lambs upon the transition from early to late gestation, indicative of increased MFB content. aSMA staining was absent in wounds generated at gestation day 75 but was present at progressively greater amounts in wounds generated at gestation day 100 or 120 (term, 145 days). Another convincing demonstration that MFB is associated with wound contraction and scar formation in fetal wounds was provided by Cass and coworkers ${ }^{16}$. This study confirmed that the healing of fetal lamb skin wounds by regeneration was gradually abolished when injury generation

${ }^{1}$ Department of Mechanical Engineering, Massachusetts Institute of Technology, Cambridge, MA, USA. ${ }^{2}$ Department of Mechanical and Manufacturing Engineering, University of Cyprus, Nicosia, Cyprus. ${ }^{\circledR}$ email: yannas@mit.edu 
was delayed from gestational day 60-90, while simultaneously scar formation progressively dominated wound healing. The observations of Estes et al. and Cass et al. can be explained by the simple hypothesis that gestation age affects, through some yet unknown mechanism, MFB differentiation, which in turn switches the mode of fetal skin wound healing from regeneration to scar formation.

\section{SCAR FORMATION IS A KEY OUTCOME OF WOUND CONTRACTION}

Scar synthesis, the hallmark of failed regeneration in injured skin, has been widely studied in adult mammals; in contrast, the study of wound contraction has been relatively neglected. In a contracting adult skin wound, large numbers of MFBs are held together in assemblies by intercellular junctions ${ }^{13}$. The organization of such MFB assemblies, particularly the spatial orientation of individual MFB axes, appear to be aligned with the physiological macroscopic wound contraction forces that eventually reduce wound size ${ }^{17,18}$. The spatial distribution of MFB assemblies and the resulting mechanical stress field generated in the injury site due to the contractile forces applied by MFB depend on the anatomical shape of the injured organ and the local geometry of the wound undergoing healing. For example, skin wounds (planar geometry) in rodents close by stresses aligned along the epidermal plane; wounded stumps that result from transection of the rat sciatic nerve (cylindrical geometry) close by circumferential compressive stresses (hoop stresses) (Fig. 1b, g) ${ }^{19,20}$. It has been shown that collagen-synthesizing cells, such as fibroblasts and MFBs, deposit newly-synthesized collagen fibers along a direction approximately parallel to their own axes ${ }^{21}$. Accordingly, the average orientation of collagen fibers synthesized during spontaneous wound healing coincides with the major direction of the mechanical stress field generated by highly-organized MFB clusters, leading to the synthesis of a highly anisotropic fibrous tissue (scar) ${ }^{22-24}$. Furthermore, experiments with full-thickness skin wounds in the guinea pig $^{18}$ and in the transected rat sciatic nerve ${ }^{19}$ have shown that wound contraction precedes scar formation by several days ${ }^{11}$.

We hypothesize that scar formation is driven by wound contraction according to the following sequence: Tissue injury generates a wound, which normally closes by a mechanical contraction force. This force acts on the fluid and cellular contents of the wound, orienting the axes of myofibroblasts, as shown in the data for wounds for three injured organs (Fig. 1b, d, g, i, l, and $\mathrm{n})$. Since it has been shown that fibroblasts synthesize collagen fibrils with fiber axes that are parallel to the long axis of the synthesizing cells ${ }^{21}$, oriented fibroblasts in the wound bed are expected to synthesize collagen fibers that are likewise oriented along the same macroscopic axis. This process leads to the formation of scar tissue, a bundle of highly aligned collagen fibers, as previously observed by scanning electron microscopy ${ }^{22}$ and laser light scattering ${ }^{23}$. Clearly, this hypothetical mechanism relies on the effect of a mechanical force and neglects other factors, such as hypothetical regulation of the inflammatory response, e.g. by modulation of the immune cell milieu, that modifies healing to yield scarring. Previous studies have demonstrated the effect of mechanical forces on collagen synthesis by fibroblasts, similar to the forces discussed here ${ }^{25}$.

\section{BLOCKING OF WOUND CONTRACTION BY DRT}

A simple and direct test of the hypothesis that wound contraction is associated with scar formation consists of using an efficient contraction-blocker and observing if such use leads to the prevention of scars. Dermis regeneration template (DRT), a highly porous collagen-based scaffold, has been shown to be effective in blocking contraction in skin wounds ${ }^{26}$, peripheral nerve wounds ${ }^{20}$, and the wounded conjunctival stroma ${ }^{27}$.
The effect of DRT on MFB morphology is profound. Blocking of the macroscopic wound contraction force by DRT is associated with the combined effect of a sharp decrease in MFB density, dispersion of MFB assemblies, and disorientation of MFB long axes. The decrease in MFB density by DRT, down to $20 \%$ of untreated controls ${ }^{18}$, appears to follow from the observed DRTinduced downregulation of the inflammatory response ${ }^{20}$, including cytokines required for MFB differentiation such as TGF $\beta 1^{12}$. Dispersion of MFB assemblies and disorientation of MFB long axes is associated with extensive binding of MFB on the DRT surface ${ }^{28}$. Extensive binding of this sort requires a sufficiently high magnitude of specific surface area inside the porous scaffold, a requirement which is satisfied by a small average pore diameter, about $100 \mu \mathrm{m}$ in an optimized scaffold ${ }^{26}$.

The surface chemistry (density of ligands for specific integrins) of collagen scaffolds is pivotal for the DRT-MFB binding interaction. It has been shown that MFB cannot attach on collagen scaffolds whose integrin ligands have been chemically modified $^{28}$. Integrin-ligand binding in DRT-treated wounds apparently changes the MFB-MFB binding mode characteristic of MFB assemblies in normally contracting wounds to a mostly MFBDRT binding mode which keeps MFB apart from each other and disrupts MFB assemblies. Modification of the contractile phenotype is eventually followed by blocking of scar and incidence of regeneration (see below).

A detailed description of methods for preparing DRT scaffolds suitable for grafting wounds in skin and peripheral nerves, as well as further details on the mechanism for DRT activity, have appeared in a monograph ${ }^{29}$.

\section{BLOCKING WOUND CONTRACTION IN THREE ADULT MAMMALIAN SPECIES LEADS TO PARTIAL REGENERATION}

Direct support of the hypothesis that inability to regenerate injured adult organs is associated with the presence of wound contraction is provided in a series of experimental studies, which focused on the property of DRT to block wound contraction and induce regeneration in three models of adult mammalian injury: fully-excised guinea pig $\operatorname{skin}^{11,18,26,30}$, transected rat sciatic nerve $^{19,20,31}$, and fully-excised conjunctival stroma ${ }^{27}$.

During spontaneous healing (no DRT grafting) the spatial morphology of MFB in the three injured sites differed in apparent deference to the anatomical wound shape which required closure. MFB was organized in a thick planar layer (skin, conjunctiva) or a circumferential layer (peripheral nerves) of dense highly-organized MFB cells suggesting the occurrence of significant wound contraction that had adapted to the shape of the specific injury and organ (Fig. 1b, g, and I).

In all three animal models, spontaneous healing (no DRT grafting) after severe injury led to scar formation (Fig. 1c, h, and m). Specifically, spontaneous healing of full-thickness skin wounds in the guinea pig led to the formation of highly oriented collagen fibers in the plane of the epidermis, characteristic of dermal scar (Fig. 1C) ${ }^{30}$ nerve fibers in the transected rat sciatic nerve trunk lost their characteristic structure and presented instead of a sheaf of collagen fibers, identified as a neural scar (Fig. 1h) ${ }^{32}$ excisions of the conjunctival stroma in a rabbit led to the formation of fibrous tissue, identified as a scar, that was highly oriented in the plane of the stroma (Fig. $1 \mathrm{~m})^{27}$.

In contrast, in all three models, grafting the injury site with DRT greatly reduced the number of MFBs at the injury site, dispersed MFB assemblies, and disoriented MFB axes (Fig. 1d, i, and n). As detailed in the preceding section, each of these MFB phenotypic changes observed in the presence of DRT has been associated with the observed abolition of macroscopic wound contraction. In all three animal models, grafting the injury site with DRT led to the synthesis of physiological or nearly physiological new tissue (Fig. 1e, $\mathrm{j}$, and o). Indeed, the histomorphometric and electrophysiological 
Injury

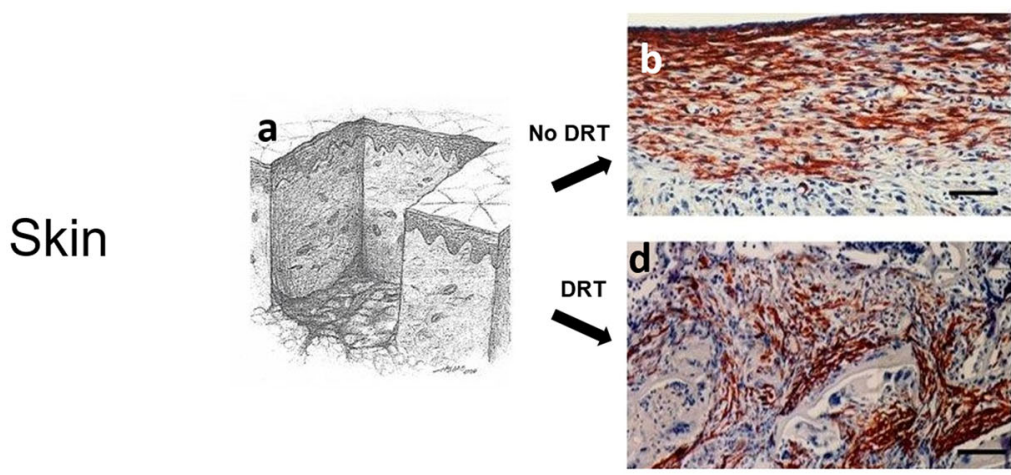

Tissue
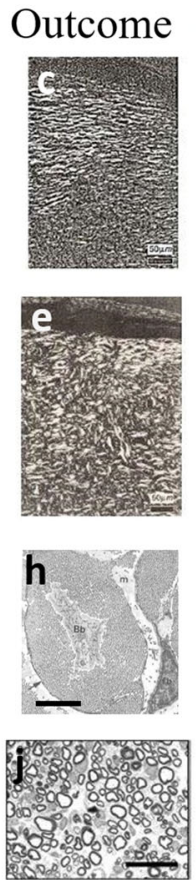

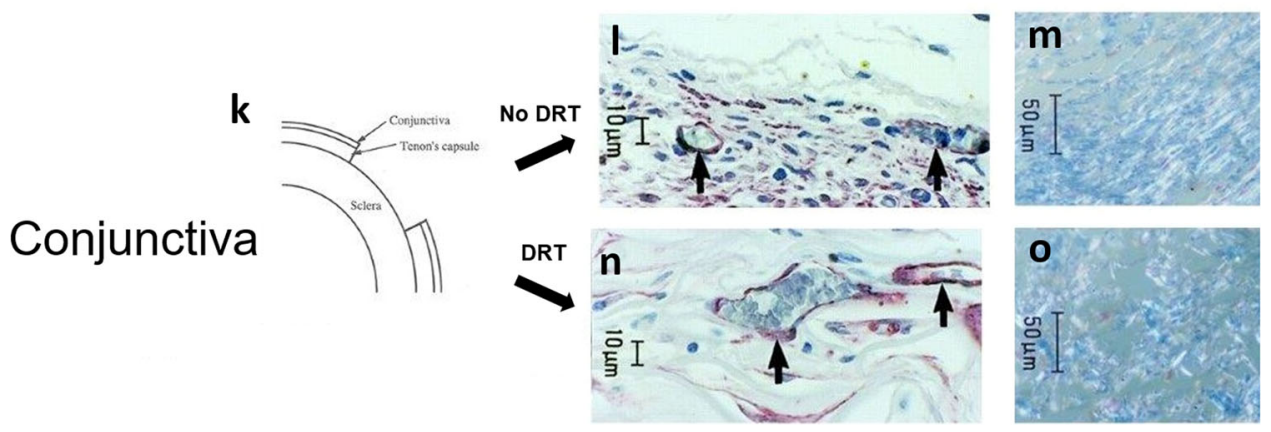

Fig. 1 Wound contraction and associated wound healing response in three adult injury models in the absence (spontaneous healing) and presence of a DRT graft. a-e guinea pig full-thickness excised skin wound. $\mathbf{f}-\mathbf{j}$ fully transected rat sciatic nerve. $\mathbf{k}-\mathbf{o}$ excised rabbit conjunctival stroma. $\mathbf{a}, \mathbf{f}$, and $\mathbf{k}$ Schematic of the corresponding injury site model. $\mathbf{b}, \mathbf{d}, \mathbf{g}$, $\mathbf{i}$, $\mathbf{l}$, and $\mathbf{n}$ Immunohistochemical localization of $\alpha \mathrm{SMA}^{+}$myofibroblasts (red brown) 10 days after skin injury $(\mathbf{b}, \mathbf{d})^{20}, 7$ days after peripheral nerve transection $(\mathbf{g} \text {, i })^{20}$, or 14 days after conjunctiva injury $(\mathbf{I}, \mathbf{n})^{27} . \mathbf{b}, \mathbf{g}$, and I Lack of DRT grafting or grafting with control grafts led to large, dense, highly-aligned MFB configurations. d, $\mathbf{i}$, and $\mathbf{n}$ DRT grafting led to significantly fewer dispersed, and almost randomly aligned MFBs. Scale bars: skin and nerves, $100 \mu$ m; conjunctiva, $10 \mu \mathrm{m}$. $\mathbf{c}, \mathbf{e}, \mathbf{h}, \mathbf{j}, \mathbf{m}$, and $\mathbf{o}$ Evaluating the structure of the resulting tissue. $\mathbf{c}$, e In full-thickness skin wounds birefringence microscopy of collagen fibers demonstrates the formation of scar in ungrafted wounds (c) and the synthesis of the nearly-physiological dermis in DRT-grafted wounds $(\mathbf{e})^{18,26,30}$. $\mathbf{h}$ In DRT-ungrafted peripheral nerve wounds electron microscopy reveals that 26 months following transection, the original nerve fibers have been replaced by a dense sheaf of collagen fibrils that enclose groups of Schwann cells (Büngner bands, $\mathrm{Bb})^{32}$. $\mathrm{j}$ In contrast, histological micrographs of cross-sections from DRT-grafted peripheral nerve wounds demonstrate the formation of neural tissue whose histomorphometric (equivalent diameter, number of myelinated fibers, number of A-fibers) and electrophysiological assays were similar to those for the autograft ${ }^{31} . \mathbf{m}$, o In conjunctiva wounds, immunohistochemical analysis and birefringence microcopy demonstrate scar formation in ungrafted wounds ( $\mathbf{m}$, note marked orientation of birefringent collagen fibers) and synthesis of near-normal conjunctival stroma collagen fibers (absence of orientation) in DRT-grafted wounds (o) ${ }^{27}$. Scale bars: skin, $50 \mu \mathrm{m}$; peripheral nerves, neural scar (top), $1 \mu \mathrm{m}$; peripheral nerve, regenerated nerve (bottom), $25 \mu \mathrm{m}$; conjunctiva, $50 \mu \mathrm{m}$. Figure $1 \mathrm{a}$, $\mathrm{f}$ was reproduced with permission from Springer Nature ${ }^{29}$. Fig. 1b, d, g, i, and j were reproduced with permission from Biomaterials, 33, 4783-91, ${ }^{\circ}$ Elsevier (2012). Figure 1c, e were reproduced with permission from $\mathrm{MIT}^{30}$. Figure $1 \mathrm{~h}$ was reproduced with permission from J. Anat., 192, 529-39, ${ }^{\circ}$ Wiley (1998). Figure $11-0$ was reproduced with permission from Invest. Ophthalmol. Vis. Sci, 41, 2404-11, ${ }^{\circ}$ Association for Research in Vision and Ophthalmology (2000).

properties of transected rat sciatic nerves, studied at an initial $10 \mathrm{~mm}$ gap between transected stumps that was tubulated with DRT conduits, were statistically indistinguishable from those of the autograft control at 60 weeks post injury ${ }^{31}$.

A key observation that supports a hypothetical antagonistic relationship between wound contraction and induced regeneration is the known dependence of the contraction-blocking and partially regenerating activity of DRT grafts on the specific physicochemical properties of the scaffold ${ }^{26}$. In addition to presenting to cells surface-chemical features specific for integrinligand binding ${ }^{28}$, DRT scaffolds are further characterized by optimal levels of the degradation half-life and the average pore size ${ }^{20,26}$. Deliberate synthesis of grafts with progressively suboptimal properties resulted in increasingly larger MFB capsules in peripheral nerve stumps and gradually resulted in worse wound healing outcomes that eventually inclined towards spontaneous healing. In particular, a DRT scaffold library, synthesized by systematically perturbing specific DRT properties away from their 
a
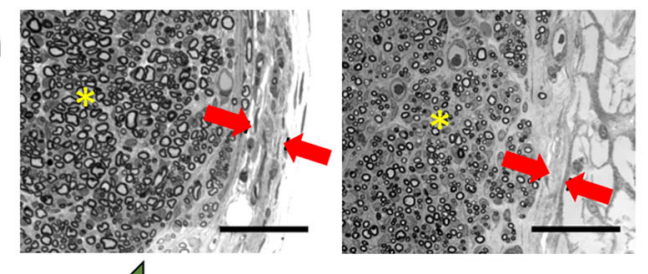
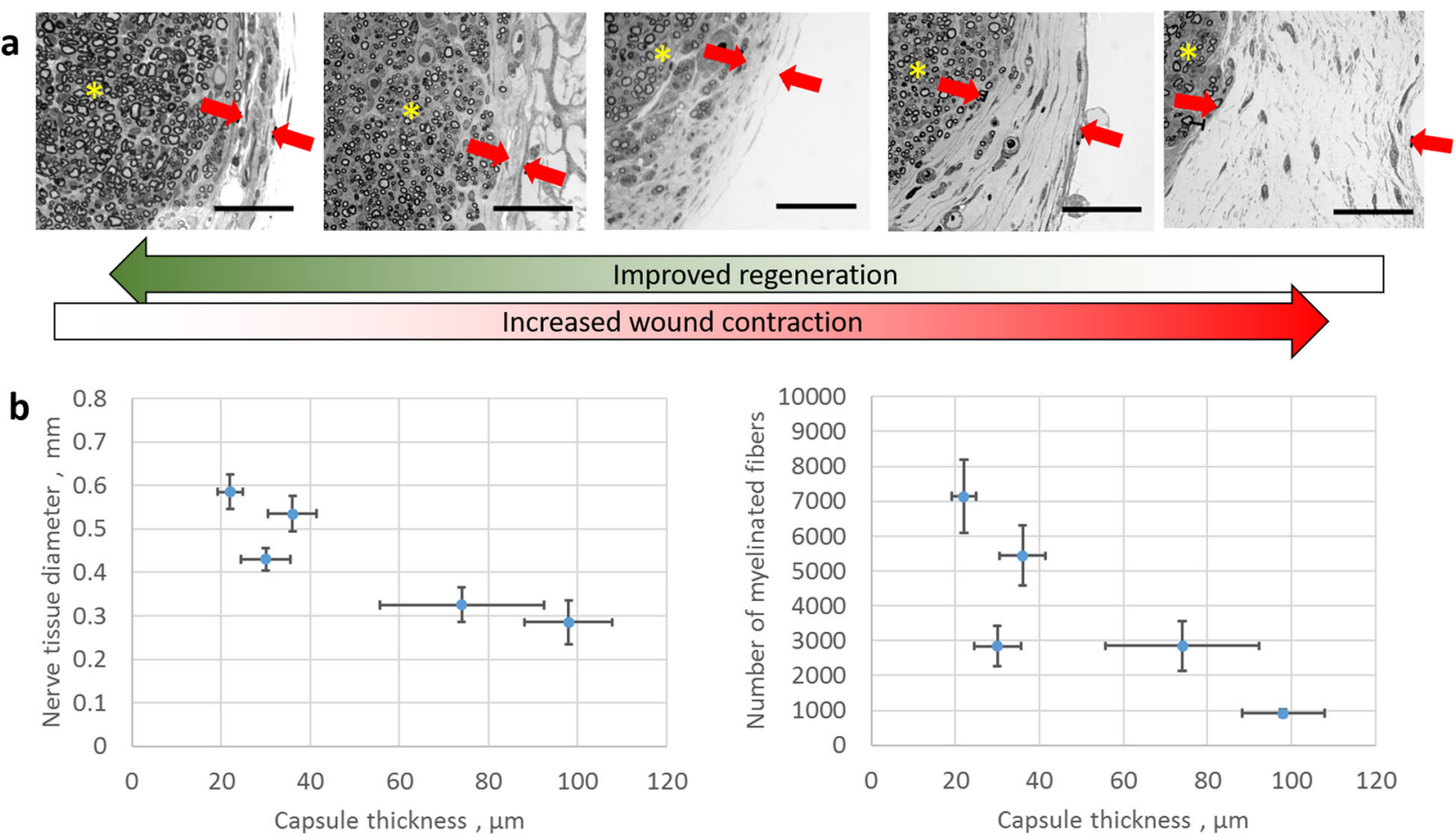

Fig. 2 An inverse relationship between wound contraction and induced regeneration was demonstrated in transected rat sciatic nerves grafted by a library of five different porous collagen scaffolds, either DRT or analogs of DRT, differing only in degradation half-life. $\mathrm{a} \mathrm{OsO}_{4}$ staining of tissue sections reveals the formation of a contractile MFB capsule (thickness shown between red arrows) around the newlyformed nerve tissue (stars) 9 weeks post-injury. b Quantification of the inverse relationship between the intensity of wound contraction (assayed by the radial thickness of the MFB capsule) and quality of induced regeneration, assayed both by the equivalent tissue diameter (left) and the number of myelinated fibers (right). Data (mean $\pm \mathrm{se}$ ) were obtained at the midpoint of the gap distance, $15 \mathrm{~mm}$, initially separating the two nerve stumps, measured 9 weeks post-injury ${ }^{20}$. Scale bars $50 \mu \mathrm{m}$. Figure $2 \mathrm{a}$, b were reproduced with permission from Biomaterials, 33 , 4783-91, ${ }^{\circ}$ Elsevier (2012).
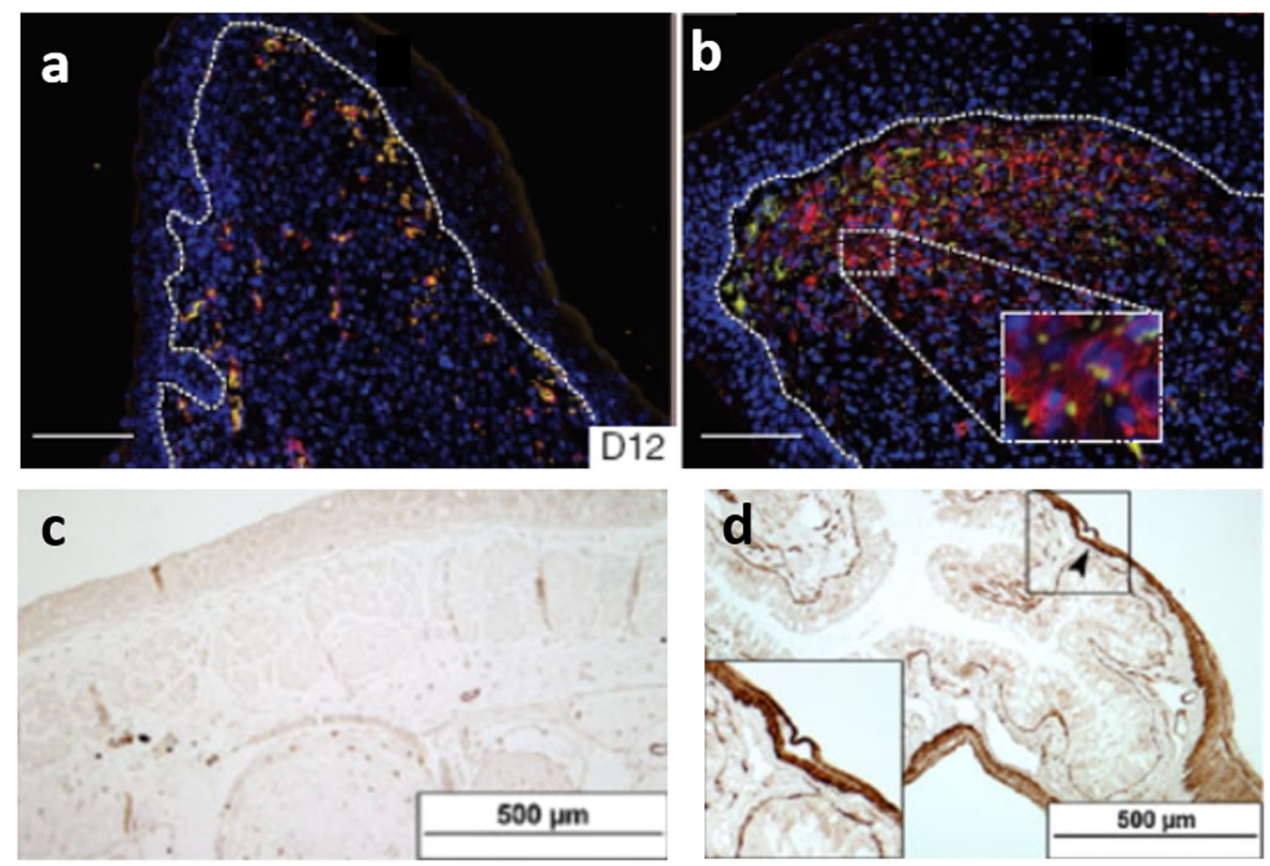

Fig. 3 The absence of wound contraction has been reported in several cases of spontaneous scarless healing in various species. a No $\alpha \mathrm{SMA}^{+}$fibroblasts (red) were detected 12 days after full-thickness ear injury in $A$. kempi, which are able to spontaneously regenerate severe skin injuries. b In contrast, a significant number of $\alpha \mathrm{SMA}^{+}$cells (red) were detected in ear injuries in Mus musculus mice, which spontaneously heal such injuries by forming $\operatorname{a~scar~}^{35}$. c, d The axolotl can regenerate spontaneously injuries in several organs, including its limbs and tail. $\mathbf{c} \alpha S M A$ staining was not detected in skin injuries, 12 days after injury. $\mathbf{d}$ In the same animal, $\alpha S M A$ (brown) was detected in control tissue (small intestine) ${ }^{33-39}$. Figure $3 a$, b were reproduced with permission from Nature $489,561-66$, ${ }^{\circ}$ Springer Nature (2012). Figure 3c, d was reproduced with permission from J. Exp. Zool. B. Mol. Dev. Evol., 314B, 684-97, ${ }^{\circ}$ Wiley (2010). 
optimal values, led to scaffolds that were gradually less active regeneratively than $\mathrm{DRT}^{20}$. Quantitative data from transected rat peripheral nerves grafted with scaffolds from such a DRT library highlight an inverse relationship between the thickness of the contractile MFB capsule surrounding the transected nerve stump (an indirect assay of wound contraction magnitude) and two metrics of the quality of the resulting nerve tissue (regenerated nerve diameter and number of myelinated axons) ${ }^{20}$, as presented in Fig. 2. The data in Fig, 2 represent direct, quantitative support of the antagonistic relation between contraction and induced regeneration of peripheral nerves.

In summary, experimental data from three adult mammalian models of severe injury (Figs. 1,2) provide multifaceted support of the hypothesis that blocking of wound contraction by DRT prevents scar formation and favors regeneration.

\section{DISCUSSION}

While the early fetus has been shown capable of regenerating severe skin wounds, the late fetus and the adult mammal spontaneously close skin wounds by contraction and scar formation rather than regeneration. Yet, the observed ability, reported here, to induce partial regeneration in three adult mammalian injury models by DRT grafts that blocked wound contraction (Figs. 1, 2) suggests that the late fetus and the adult mammal retain the ability to regenerate their injured organs if healing is properly guided.

The observation that the occurrence of wound contraction consistently leads to healing by scar formation in adult mammals supports the hypothesis that wound contraction prevents the synthesis of normal organ tissue (regeneration). This hypothesis as well as its reverse (blocking of wound contraction prevents scar formation and favors regeneration) are directly supported by the observed ability of DRT grafts to block wound contraction and its hypothetical sequel, scar formation, and instead induce regeneration in the three animal organ injury models discussed above (Fig. 1). This hypothesis is directly supported by the observed inverse relation between the thickness of the contractile cell capsule and each of two measures of quality of nerve regeneration (Fig. 2).

The unexpected association of induced regeneration with blocked wound contraction is further supported by several independent observations from diverse species. In the wellknown case of spontaneous skin regeneration in the injured rabbit ear, direct photographic observation demonstrates the absence of contraction when the skin was excised from one side of the rabbit ear ${ }^{7,33,34}$. Skin injuries in African spiny mice, shown to regenerate spontaneously, contain little aSMA immunostaining 12 days postinjury, in sharp contrast to Mus musculus mice that do not spontaneously regenerate severe skin injuries, (Fig. 3a, b) ${ }^{35}$. The absence of significant aSMA staining has been also observed in full-thickness excisional skin wounds in the axolotl, which heal spontaneously without a scar, (Fig. 3c, d) ${ }^{36-39}$. Although not proving a causal relation, these observations support the hypothesis that wound contraction and regeneration are processes that compete during the healing of adult mammalian wounds.

Dependence of the contraction-blocking ability of DRT on its physicochemical properties (pore size, degradation rate, surface chemistry) ${ }^{20,26,28}$ suggests that other therapeutic approaches (biomaterials, cells, small molecules), capable of regulating the molecular machinery of wound contraction, could presumably achieve similarly effective regenerative effects. While fetal wound healing data originate from skin injury studies, evidence from adult injury models presented above extends the effects of wound contraction blocking on regeneration beyond skin wounds to other organs where contraction is a major mechanism for wound healing.

We propose the hypothesis that mammals are apparently born with a built-in latent ability to regenerate injured organs, which remains dormant until it is activated by injury. Diverse experimental evidence supports the hypothesis that wound contraction impedes such a latent regenerative activity during spontaneous wound healing both in the late fetus and in adults. However, the ability of specific contraction-blocking grafts based on DRT to induce regeneration suggests that this hypothetical endogenous regenerative potential can be released by treatments that block the cellular and molecular origins of wound contraction. This hypothesis should affect ongoing efforts to design effective regenerative treatments of all kinds (biomaterials, small molecules, cell therapies) for injured or dysfunctional organs. Any emerging regenerative medicine treatments under investigation may fail to activate the intrinsic regenerative capacity of adult mammals unless their design considers ways to control wound contraction.

Received: 4 December 2020; Accepted: 7 July 2021; Published online: 22 July 2021

\section{REFERENCES}

1. Longaker, M. T. et al. Studies in fetal wound healing, VI. Second and early third trimester fetal wounds demonstrate rapid collagen deposition without scar formation. J. Pediatr. Surg. 25, 63-68 (1990).

2. Mast, B. A., Diegelmann, R. F., Krummel, T. M. \& Cohen, I. K. Scarless wound healing in the mammalian fetus. Surg. Gynecol. Obstet. 174, 441-451 (1992)

3. Longaker, M. T. \& Adzick, N. S. The biology of fetal wound healing: a review. Plast. Reconstr. Surg. 87, 788-798 (1991).

4. Beanes, S. R. et al. Confocal microscopic analysis of scarless repair in the fetal rat: defining the transition. Plast. Reconstr. Surg. 109, 160-170 (2002).

5. Soo, $C$. et al. Ontogenetic transition in fetal wound transforming growth factor- $\beta$ regulation correlates with collagen organization. Am. J. Path. 163, 2459-2476 (2003).

6. Gurtner, G. C., Werner, S. \& Longaker, M. T. Wound repair and regeneration. Nature 453, 314-321 (2008)

7. Joseph, J. \& Dyson, M. Tissue replacement in the rabbit's ear. Brit. J. Surg. 53, 372-380 (1966)

8. Illingworth, C. M. Trapped fingers and amputated finger tips in children. J. Pediatr. Surg. 9, 853-858 (1974).

9. Wallace, H. Vertebrate Limb Regeneration (Wiley, New York NY, 1981).

10. Pratsinis, H., Mavrogonatou, E. \& Kletsas, D. Scarless wound healing: from development to senescence. Adv. Drug Deliv. Rev. 146, 325-343 (2019).

11. Yannas, I. V., Tzeranis, D. S. \& So, P. T. C. Regeneration of injured skin and peripheral nerves requires control of wound contraction, not scar formation. Wound Repair Regen. 25, 177-191 (2017).

12. Desmoulière, A., Chaponnier, C. \& Gabbiani, G. Tissue repair, contraction, and the myofibroblast. Wound Repair Regen. 13, 7-12 (2005).

13. Hinz, B. et al. Recent developments in myofibroblast biology: paradigms for connective tissue remodeling. Am. J. Pathol. 180, 1340-1355 (2012).

14. Desmoulière, A., Redard, M., Darby, I. \& Gabbiani, G. Apoptosis mediates the decrease in cellularity during the transition between granulation tissue and scar. Am. J. Pathol. 146, 56-66 (1995).

15. Estes, J. M. et al. Phenotypic and functional features of myofibroblasts in sheep fetal wounds. Differentiation 56, 173-181 (1994).

16. Cass, D. L., Sylvester, K. G., Yang, E. Y., Crombleholme, T. M. \& Adzik, N. S. Myofibroblast persistence in fetal sheep wounds is associated with scar formation. $J$. Pediatr. Surg. 32, 1017-1021 (1997).

17. Baur, P. S. Jr., Parks, D. H. \& Hudson, J. D. Epithelial mediated wound contraction in experimental wounds - the purse-string effect. J. Trauma 24, 713-720 (1984).

18. Murphy, G. F., Orgill, D. P. \& Yannas, I. V. Partial dermal regeneration is induced by biodegradable collagen-glycosaminoglycan grafts. Lab. Invest. 62, 305-313 (1990).

19. Chamberlain, L. J., Yannas, I. V., Hsu, H. P. \& Spector, M. Connective tissue response to tubular implants for peripheral nerve regeneration: the role of myofibroblasts. J. Comp. Neurol. 417, 415-430 (2004).

20. Soller, E. C., Tzeranis, D. S., Miu, K., So, P. T. \& Yannas, I. V. Common features of optimal collagen scaffolds that disrupt wound contraction and enhance regeneration both in peripheral nerves and in skin. Biomaterials 33, 4783-4791 (2012).

21. Birk, D. E. \& Trelstad, R. L. Fibroblasts compartmentalize the extracellular space to regulate and facilitate collagen fibril, bundle, and macro-aggregate formation, in Extracellular Matrix: Structure and Function (Alan R. Liss, New York NY, pp. 373-382, 1985).

22. Hunter, J. A. A. \& Finlay, J. B. Scanning electron microscopy of normal scar and keloids. Br. J. Surg. 63, 826-830 (1976). 
23. Ferdman, A. G. \& Yannas, I. V. Scattering of light from histologic sections: a new method for the analysis of connective tissue. J. Invest. Dermatol. 100, 710-716 (1993).

24. Tomasek, J. T., Gabbiani, G., Hinz, B., Chaponnier, C. \& Brown, R. A. Myofibroblasts and mechano-regulation of connective tissue remodeling. Nat. Rev. Mol. Cell Biol. 3, 349-363 (2002).

25. Rolin, G. L. et al. In vitro study of the impact of mechanical tension on the dermal fibroblast phenotype in the context of skin wound healing. J. Biomech. 47, 3555-3561 (2014)

26. Yannas, I. V., Lee, E., Orgill, D. P., Skrabut, E. M. \& Murphy, G. F. Synthesis and characterization of a model extracellular matrix which induces partial regeneration of adult mammalian skin. Proc. Natl Acad. Sci. USA 86, 933-937 (1989).

27. Hsu, W. C., Spilker, M. H., Yannas, I. V. \& Rubin, P. A. D. Inhibition of conjunctival scarring and contraction by a porous collagen-GAG implant. Invest. Ophthalmol. Vis. Sci. 41, 2404-2411 (2000).

28. Tzeranis, D. S., Soller, E. C., Buydash, M. C., So, P. T. \& Yannas, I. V. In situ quantification of surface chemistry in porous collagen biomaterials. Ann. Biomed. Eng. 44, 803-815 (2016).

29. Yannas, I. V. Tissue and Organ Regeneration in Adults. Chs. 8, 9 (2nd edition) (Springer, NY, 2015).

30. Orgill, D. P. The effects of an artificial skin on scarring and contraction in open wounds, Ph.D. thesis, Massachusetts Institute of Technology, Cambridge MA (1983).

31. Chamberlain, L. J., Yannas, I. V., Hsu, H. P., Strichartz, G. \& Spector, M. CollagenGAG substrate enhances the quality of nerve regeneration through collagen tubes up to level of autograft. Exp. Neurol. 154, 315-329 (1998).

32. Bradley, J. L., Abernethy, D. A., King, R. H., Muddle, J. R. \& Thomas, P. K. Neural architecture in transected rabbit sciatic nerve after prolonged nonreinnervation. J. Anat. 192, 529-538 (1998)

33. Goss, R. J. \& Grimes, L. N. Tissue interactions in the regeneration of rabbit ear holes. Am. Zool. 12, 151-157 (1972).

34. Mustoe, T. A., Pierce, G. F., Morishima, C. \& Deuel, T. F. Growth factor-induced acceleration of tissue repair through direct and inductive activities in a rabbit dermal ulcer model. J. Clin. Invest 87, 694-703 (1991).

35. Seifert, A. W. et al. Skin shedding and tissue regeneration in African spiny mice (Acomys). Nature 489, 561-565 (2012).

36. Seifert, A. W., Monaghan, J. R., Voss, S. R. \& Maden, M. Skin regeneration in adult axolotls: a blueprint for scar-free healing in vertebrates. PLoS One 7, e32875 (2009).

37. Levesque, M. et al. Transforming growth factor beta signaling is essential for limb regeneration in axolotls. PloS ONE 2, e1227-e1228 (2012).

38. Denis, J. F., Lévesque, M., Tran, S. D., Camarda, A. J. \& Roy, S. Axolotl as a model to study scarless wound healing in vertebrates: role of the transforming growth factor beta signaling pathway. Adv. Wound Care 2, 250-260 (2013).
39. Lévesque, M., Villiard, E. \& Roy, S. Skin wound healing in axolotls: a scarless process. J. Exp. Zool. B Mol. Dev. Evol. 314, 684-697 (2010).

\section{ACKNOWLEDGEMENTS}

IVY acknowledges partial support by grant RO1 NS051320 from the National Institutes of Health.

\section{AUTHOR CONTRIBUTIONS}

I.V.Y. and D.S.T. wrote the paper.

\section{COMPETING INTERESTS}

The authors declare no competing interests.

\section{ADDITIONAL INFORMATION}

Correspondence and requests for materials should be addressed to I.V.Y.

Reprints and permission information is available at http://www.nature.com/ reprints

Publisher's note Springer Nature remains neutral with regard to jurisdictional claims in published maps and institutional affiliations.

(i) Open Access This article is licensed under a Creative Commons cc) Attribution 4.0 International License, which permits use, sharing, adaptation, distribution and reproduction in any medium or format, as long as you give appropriate credit to the original author(s) and the source, provide a link to the Creative Commons license, and indicate if changes were made. The images or other third party material in this article are included in the article's Creative Commons license, unless indicated otherwise in a credit line to the material. If material is not included in the article's Creative Commons license and your intended use is not permitted by statutory regulation or exceeds the permitted use, you will need to obtain permission directly from the copyright holder. To view a copy of this license, visit http://creativecommons. org/licenses/by/4.0/.

(c) The Author(s) 2021 